\title{
TRICHODERMA SPP ANTAGONISMO A FITOPATÓGENOS CAUSANDO PUDRICIÓN DE RAÍZ DE YUCA EN ALAGOAS
}

\section{ARTÍCULO DE REVISIÓN}

SILVA, Izael Oliveira ${ }^{1}$

AMORIM, Edna Peixoto da Rocha ${ }^{2}$

JUNIOR, Nelson Augusto Nascimento ${ }^{3}$

PEIXINHO, Georgia Souza ${ }^{4}$

CARNAÚBA, Juliana Paiva ${ }^{5}$

NETO, Vicente Ferreira de Araujo ${ }^{6}$

SILVA, Izael Oliveira. Et al. Trichoderma spp antagonismo a fitopatógenos causando pudrición de raíz de yuca en alagoas. Revista Científica Multidisciplinar Núcleo do Conhecimento. Año 05, Ed. 06, Vol. 12, págs. 149-177. Junio de 2020. ISSN: 2448-0959, enlace

de acceso:

https://www.nucleodoconhecimento.com.br/agronomia/antagonismo-de-trichoderma

${ }^{1}$ Doctor en Protección Fitosanitaria, Maestría en Producción Vegetal, Licenciatura y Licenciatura en Ciencias Biológicas.

2 Doctor en Agronomía (Agricultura). Máster en Fitopatología. Especialización en Didáctica para la Educación Superior. Graduación en Agronomía.

3 Doctor en Protección Fitosanitaria. Máster en Agronomía (Irrigación y Drenaje). Graduación en Agronomía.

${ }^{4}$ Máster en Horticultura De Regadío. Graduación en Agronomía.

${ }^{5}$ Doctor en Fitopatología. Máster en Agronomía (Producción Vegetal y Protección Vegetal). Mejora en la Mejora de las Ciencias Biológicas. Graduación en Agronomía.

${ }^{6}$ Graduado en Agronomía. 


\section{RESUMEN}

Varios son fitopatógenos que limitan la producción de yuca (Manihot esculata Crants) siendo la podredumbre de las raíces a las que se casan con la mayor pérdida en el cultivo alcanzando el $70 \%$. Esta cultura es de gran interés para la región noreste de Brasil, proporcionando grandes pérdidas. Las dificultades de gestión han dirigido la búsqueda de alternativas, entre las que, los biocontroladores han mostrado resultados significativos. Esta revisión de la literatura tenía como objetivo recopilar información relacionada con la podredumbre de raíz de yuca cv Rosinha, los principales fitopatógenos y actividades antagónicas de Trichoderma en el control de enfermedades en el estado de Alagoas. Se realizó una encuesta bibliográfica en las bases de datos Scielo, Google Acad-mico, portal de Capes (tesis y disertaciones), revistas y libros. Para obtener información sobre: pudrición de la raíz de la yuca de mesa, Trichoderma spp., como un agente de biocontrol de fitopatógenos.

Palabras clave: Raíz tuberosa, control biológico, fitopatología.

\section{INTRODUCCIÓN}

Debido a su valor nutricional, Manihot esculenta Crantz destaca en Brasil y en el mundo con una importante contribución económica y social debido a su versatilidad, ya que se utiliza tanto en alimentos animales como humanos y ampliamente utilizado en la industria. El cultivo ejerce un apoyo significativo a la población con bajo poder adquisitivo, ya que además de ser una fuente nutritiva también es un generador de ingresos en la agricultura familiar (NOTARO et al. 2013).

En el mundo, el consumo de yuca y sus derivados se encuentran en la sexta posición en el ranking de soja, trigo, arroz, maíz y patatas los cultivos que ocupan las primeras posiciones según datos de la fao (2012). Brasil es el cuarto productor más grande del mundo, con una cosecha estimada para 2016 de 25 millones de toneladas (IBGE, 2015). 
De gran versatilidad, el cultivo se utiliza plenamente, no sólo la parte aérea (hojas y tallos) sino también la raíz. Las hojas tienen su uso en alimentos humanos (suplemento) y animales (aplastados). Los tallos, en la alimentación animal, en forma de silages y heno y todavía en natura. La raíz, en la alimentación humana, animal y también por industrias, principalmente harina, almidones, plásticos biodegradables, productos textiles y biocombustibles (CONAB, 2013).

El cultivo de yuca se enfrenta a muchos desafíos, incluidos los problemas fitosanitarios (NASCIMENTO J-NIR, 2015). Varias enfermedades ya se han reportado en el cultivo, entre ellas, podredumbre de raíz, cuyos agentes pueden ser Lasiodiplodia sp., Pythium sp., Rosellinia sp., Fusarium sp., Neoscytalidium lignicola, Phytophthora drechsleri Tucker sin embargo, los últimos tres fitopatógenos son los más frecuentemente involucrados en la raíz de pudrición en el estado de Alagoas (Figura 1) y en la mayoría de las regiones productoras. La información sobre la podredumbre de las raíces de yuca sigue siendo incipiente y es de suma importancia para servir como herramienta en la toma de decisiones sobre la mejor estrategia a utilizar (MASSOLA JR; BEDENDO, 2005; MUNIZ et al., 2006; NOTARO et al., 2013; SILVA, 2013; FUKUDA, NO VOY A SER OTSUBO, 2015).

Entre los microorganismos que causan pudrición de raíz de yuca $P$. drechsleri y $F$. solani, son los más importantes debido al alcance y la gravedad causando pérdidas significativas en la producción. En suelos densos y acidificados la ocurrencia de Fusarium sp., es más frecuente, y una de las características de estos patógenos es causar daños en el tallo cerca del suelo por infección de los vasos xilémicos y consecuentemente interferir en la circulación de la savia, causando así la podredumbre indirecta de la raíz en cualquier causa de desarrollo vegetal. A diferencia de Phytophthora, cuyos síntomas provocados en las raíces son de color marrón y pudrición de consistencia suave que exudan un líquido con mal olor, ya las pudriciones de Fusarium sp., sin alteraciones aparentes en los tejidos con signos de deshidratación y aparición momificada de una podredumbre seca (MOURA; SILVA, 1997; POLTRONIERI et al., 2001; SILVA, no voy a TEIXEIRAS, 2012). 
Como medidas de protección y control de la podredumbre de las raíces, se utiliza la gestión física y química del suelo, pero se ha apoyado positivamente el uso de variedades tolerantes asociadas a prácticas culturales como la rotación de cultivos y el sistema de cultivo. Nascimento Júnior (2015), destaca que es de fundamental importancia conocer las condiciones edapho-climáticas de la región de cultivo y el uso de técnicas apropiadas que puedan ser adoptadas por el agricultor, para tener un retorno económico dentro de un sistema agrícola sostenible. El control alternativo de fitopatógenos con antagonistas, especialmente especies de trichoderma, ya ha sido estudiado, debido al interés mundial de utilizar productos menos agresivos con el medio ambiente (SOUSA et al., 2012; SILVA et al., 2014). Para Robbs (1992) y Barbosa; Meza (2009) la competencia por la alimentación y la antibiosis, son los mecanismos más utilizados por algunas especies de Trichoderma, siendo considerado por lo tanto un excelente agente de biocontrol.

En vista de lo anterior, el objetivo del presente trabajo era realizar una revisión bibliográfica sobre los principales fitopatógenos y el potencial del antagonismo de Trichoderma implicado con la podredumbre de la raíz de yuca en el estado de Alagoas.

\section{METODOLOGÍA}

El presente estudio consiste en una revisión de la literatura, realizada entre diciembre de 2014 y marzo de 2016, en la que se realizó una consulta a artículos científicos, seleccionada a través de la búsqueda en la base de datos de Google académico, LILACS, MEDLINE, SciELO, portal de Cabos (tesis y disertaciones), revistas y libros. De los numerosos artículos relacionados, 84 de ellos fueron utilizados. Como descriptores utilizamos: podredumbre de la raíz, control biológico de fitopatógenos, fitosanidad. 


\section{REVISIÓN DE LA LITERATURA}

\subsection{CULTIVO DE YUCA}

Cassava, una cosecha de gran interés para el noreste brasileño, en particular para el estado de Alagoas, es una planta perenne, arbustiva, perteneciente a la familia Euphorbiaceous, rica en almidón, utilizada en alimentos humanos y animales o como materia prima para diversas industrias (FRAIRE FILHO; BAHIA, 2015).

Nascimento Júnior (2015), destaca las ventajas de este cultivo, por su rusticidad, bajo coste, adaptación en relación con el clima, el suelo y sus características nutricionales que están relacionadas con la gran acumulación de almidón en sus raíces, así como proteínas y aminoácidos en las hojas. Es una planta originaria de las regiones tropicales de América del Sur, pero que tuvo como lugares de su domesticación el Sudeste Asiático, Africa y América Tropical (NOTARO et al., 2013; SILVA, 2013; HIJO FRAIRE; BAHIA, 2015).

En 2013, se produjeron 281.718.000 toneladas de yuca en todo el mundo, siendo Brasil el cuarto productor más grande del mundo con 23.977.757 toneladas, por detrás de Nigeria, Indonesia y Tailandia. Las regiones norte y noreste de Brasil tienen un gran volumen de producción y el área cultivada se encuentra en el sur, sureste y medio oeste, las que tienen el mayor rendimiento agrícola. El Estado de Pará es el mayor productor nacional de raíz de yuca, seguido de Paraná, Maranhao y Bahía (IBGE, 2015).

En Alagoas, las regiones que destacan como productores de yuca son la naturaleza, con el $70 \%$ de la producción total del estado (Arapiraca, San Sebastián y Campo Alegre), la zona forestal y la costa norte, siendo la primera región, la que cuenta con el mayor número de productores, responsable de 13,36 tha-1 y 240.448 toneladas, ocupando el segundo lugar en productividad en comparación con los otros estados del noreste (CONAB 2013). 
Notaro (2012) destacó que este cultivo sigue siendo de cultivo familiar y se desarrolla de manera rudimentaria, y el principal medio de propagación es vegetativo y se siguen cultivando variedades de baja calidad genética. Su utilidad se asocia con el contenido de ácido hidrocínico $(\mathrm{HCN})$ presente en hojas y raíces, lo que lo clasifica como manso o dulce (<180 mg kg-1 de HCN), también conocida como mandioca, utilizada para consumo fresco; intermedio (180 - $300 \mathrm{mg} \mathrm{kg}^{-1}$ ) y amargado o enojado (>300 mg kg1) estos dos últimos se utilizan para la alimentación animal, la producción de almidón, harina y biocombustible (OLIVEIRA et al., 2012).

La mayor utilidad de la yuca está en la industria alimentaria, principalmente en la producción de almidón destinado al mercado de almidón modificado, que además de servir cocina regional en panes, tapiocas, beijus, golosinas y pies de tomboy; es uno de los productos derivados de la planta que emplea más mano de obra y añade un alto valor en el producto procesado, según Nascimento Júnior (2015). En cuanto al uso para la extracción de productos fermentados en la industria de biocombustibles, se ha demostrado que la yuca es una buena alternativa en la fabricación de etanol, ya que presentaba un menor coste energético en la producción, comparando caña de azúcar y maíz, según Salla et al., (2010).

Según Alves (2006), la fenología de la yuca puede variar de 6 a 24 meses de cultivo, pero comercialmente es de 12 a 18 meses para el procesamiento de harina y almidón y entre 8 y 10 meses para la mesa. Entre las variedades plantadas en Alagoas, destaca la rosinha, cuyo ciclo varía entre 9 y 10 meses en tierra seca, y se puede prever de 7 a 8 meses en condiciones de sistema de regadío. Esta variedad puede alcanzar los tres metros de altura, mantiene el soporte de la planta al final del cultivo con buena producción de ramas y productividad en 21 t ha-1 (DINIZ et al., 2009).

Nascimento Júnior (2015), informa en su obra que incluso con la rusticidad del cultivo es necesario está atento a las condiciones adafo-climáticas, afirmando que la yuca produce bien en suelos fértiles, con pH entre 5.5 a 7.0 con arena o clase textural media y buen drenaje natural. La condición climática ideal se encuentra en el rango de temperatura entre $20 \mathrm{oC}$ y $27 \mathrm{oC}$, y se puede establecer en ambientes con 
temperaturas entre $160 \mathrm{C}$ y 380C; con precipitación entre 1000 y 1500 mm año-1, en regiones semiáridas entre 500 y $700 \mathrm{~mm}$ año-1; altitudes de 600 a 800 metros sobre el nivel del mar y período de luz de 12 horas día-1 (SILVA; ANDRADE, 2011; SILVA, 2013).

\subsection{ENFERMEDADES EN EL CULTIVO DE YUCA}

Varios factores pueden limitar la producción de yuca, como la aparición de plagas de insectos y fitopatógenos causantes de enfermedades, lo que afecta la calidad y el desarrollo del cultivo en Brasil y en todo el mundo. Según Nascimento Júnior (2015), la yuca es susceptible a varios fitopatógenos y esto causa graves pérdidas económicas en la producción.

Entre las enfermedades de importancia destacan las causas de lesiones en las hojas como mancha marrón (Cercosporidium henningsii) mancha blanca (Phaeoramularia manihotis), hojas ardientes (Cercospora vicosae), mancha negra (C. manihobaea) y antracnosis (Colletotrichum gloeosporioides f.sp. manihotis) que limitan los fotoasimilatos (SILVA; ANDRADE, 2011; MORAIS et al., 2013; OLIVEIRA et al., 2013). Y podredumbre de la raíz, causada por hongos y oomicetos, como Lasiodiplodia sp., Pythium sp., Rosellinia sp., Fusarium sp., Neoscytalidium lignicola, Phytophthora drechsleri Tucker sin embargo, los últimos tres fitopatógenos son los más frecuentemente involucrados en la pudrición de la raíz en el estado de Alagoas (Figura 1) y en la mayoría de las regiones productoras. La información sobre la podredumbre de las raíces de yuca sigue siendo incipiente y es de suma importancia para servir como herramienta en la toma de decisiones sobre la mejor estrategia a utilizar (MASSOLA JR; BEDENDO, 2005; MUNIZ et al., 2006; NOTARO et al., 2013; SILVA, 2013; FUKUDA, NO VOY A SER OTSUBO, 2015).

Notaro et al., (2013) y Silva (2013) advierten sobre los altos impactos socioeconómicos causados por la podredumbre de la raíz de yuca en los estados de Maranhao, Pará, Alagoas y Pernambuco, ya que está causando pérdidas significativas en la productividad, además de hacer que las áreas sean inviables para la siembra a 
lo largo de los ciclos de cultivo. Según Nascimento Júnior (2015), siendo Omyceto Phytophthora sp y el hongo Fusarium sp., son los principales patógenos del cultivo en el noreste y causan pérdidas que oscilan entre el 30 y el $70 \%$. El autor también afirma que en condiciones favorables la enfermedad puede causar $100 \%$ pérdida.

Figura 1. Aspecto de las colonias y estructuras reproductivas de los aislados de pudrición de yuca causantes de fitopatógenos en el estado de Alagoas: Fusarium spusarium. (A1-A6), Neoscytalidium sp. (B1-B6) y Phytophthora sp. (C1-C6). Colonia de Fusarium sp. (A1); conidia (A2); células conidiogénicas (A3-A6). Colonia Neoscytalidium sp. (B1); Conidia (B2-B6). Phytophthora sp colony (C1); Spomangio (C2); Chlamydospores (C3 y C4); Spomangios (C5) y Sporantia en reproducción (C6).

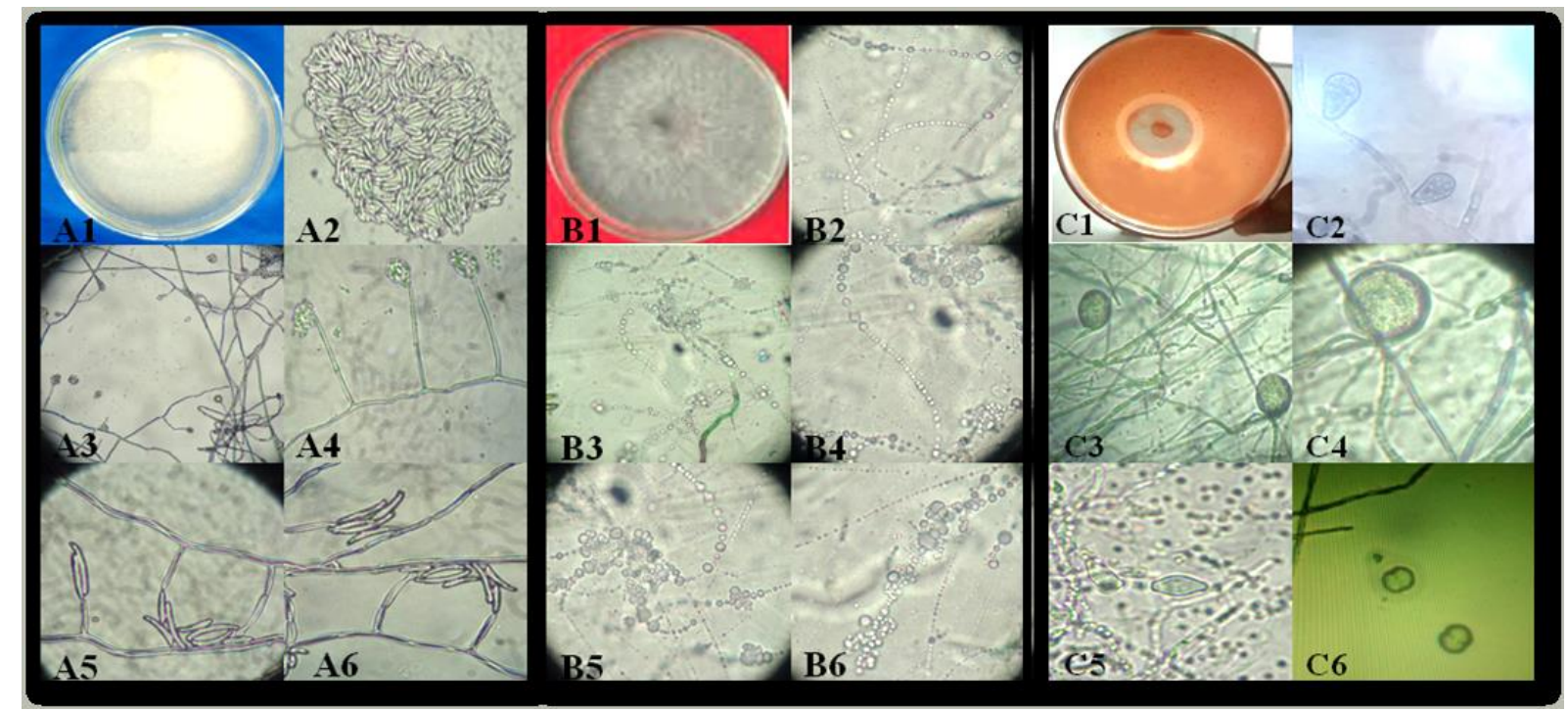

Fonte: SILVA e NASCIMENTO JÚNIOR (2016).

La podredumbre causada por Fusarium sp., se produce en plantas jóvenes y adultas, con síntomas de marchitamiento repentino y defoliación severa, que puede ser potenciada en áreas de suelo arcilloso y mal drenado (SILVA; TEXEIRA, 2012). Este hecho ya ha sido reportado por Gomes; Leal (2003), quien cuando se trabaja con yuca en los estados de Sergipe, Bahía y Alagoas, afirma que la podredumbre de la raíz es la enfermedad más limitante del cultivo en la región noreste, especialmente cuando se implanta en áreas formadas por suelo compactado.

Disponível em: https://www.nucleodoconhecimento.com.br/agronomia-es/antagonismo- 
Los fitopatógenos que causan la podredumbre tienen síntomas distintos. Los autores informan que phytophthora sp. generalmente ataca las plantas adultas, causando pudrición "suave" en las raíces, con olor muy fuerte, similar al de la materia orgánica en descomposición y la coloración grisácea. La aparición de signos es más frecuente y visible en las raíces, pero hay informes de la existencia de jóvenes que presentan síntomas en la base de los tallos de las plantas recién hermanadas, causando marchitamiento y muerte total. En el caso de la podredumbre seca causada por Fusarium sp., el daño directo de la raíz rara vez ocurre, pero el problema se acentúa en cualquier etapa del desarrollo de la planta. (GOMES; LEAL, 2003; SILVA; TEXEIRA, 2012; NASCIMENTO JÚNIOR, 2015).

\subsection{EL FUSARIUM FITOPATHOGEN}

El género Fusarium fue descrito en 1809 por Link. Los representantes de este género pertenecen al reino Fungi, phylum Ascomycota, clases Eauscomycetes y Pyrenomycetes, ordenan Hypocreales, presentando 65 especies, 55 variedades y 22 formas especiales, según Wollenweber; Reinking (1935) ${ }^{1}$ citado por Ventura (1999). Es uno de los fitopatógenos más importantes del mundo, con amplia distribución geográfica, distribuido en el suelo y en asociación con las plantas. Tener especies cosmopolitas y otras con ocurrencia restringida a ciertos ambientes (VENTURA, 1999). La mayoría de las especies son saprobicas, pero hay representantes del complejo Fusarium que producen micotoxinas que pueden afectar la salud humana y animal (URBEN et al. 2009). Seifert (2006); Querales (2010) informa que el género Fusarium es un grupo complejo que crece en número de especies y genera controversias taxonómicas para fitopatólogos.

Milanesi (2009), comenta que Fusarium sp., puede ocurrir especialmente en lugares de climas tropicales y subtropicales, y tiene una gran capacidad para sobrevivir durante largos períodos en el suelo fuera de sus huéspedes, a través de la formación de estructuras de resistencia llamadas clamidosporas. Tres grupos dentro del género son los ejemplos más específicos de estos cambios, nombrados en honor al grupo 
teleomorfo del complejo Gibberella fujikuroi (Sawada) Wollenw, el Fusarium solani (Mart.) Sacc., y el complejo F. graminearum.

El género puede presentar colonias de colores que van desde violeta, púrpura oscura a naranja, pero el predominio es de colonia pálida de rápido crecimiento, micelio aireado y difuso (MARTINS, 2005). Puhalla (1981); Martins (2005); Agrios (2005), informa que las estructuras de conidia (micro y macro), morfología, disposición, variedad de conidioforos y clamidiosporas fueron la base para la identificación de las especies de Fusarium sp. Los taxonomistas clásicos describen la presencia de microconidia unicelular y no inucleada y macroconidia multicelular frecuente con células con un solo núcleo como progenitor genéticamente idéntico. Las etapas sexuales de Fusarium son ascomicetes; la espora sexual es la ascospora. Los asignados en el género Nectria tienen ascosporas bicelulares.

Cada célula del ascopoore bicelular no está inucleada y ambos núcleos son genéticamente idénticos. Otros, como es el caso de Gibberella, forman ascosporas multicelulares. Martins (2005) menciona que las características morfológicas están influenciadas por el medio ambiente y la nutrición, por lo que los medios de cultivo se utilizan para seleccionar microorganismos y agruparlos, en función de las necesidades nutricionales que se ponen a disposición en el entorno de cultivo en condiciones estandarizadas para identificarlos. Debido a esta plasticidad y variaciones en las características fenotípicas que se encuentran en este hongo, la taxonomía basada sólo en conceptos morfológicos no es confiable. (MARTINS, 2005; URBENS et al., 2009; QUERALES, 2010).

La identificación de especies de fusarium es uno de los primeros pasos para su estudio. Tradicionalmente se ha llevado a cabo sobre la base de la morfología, desde la publicación del tratado del género "Die Fusarien" en 1935 por Wollenweber; Reinkin ${ }^{9}$. Se propusieron otros sistemas, pero no resolvieron el problema de la identificación de especies. Se incrementaron las herramientas de identificación e incluyen el uso de microscopía óptica y electrónica, medios selectivos y diferenciales, comparaciones enzimáticas, metabolitos secundarios, así como el uso de tecnologías 
inmunológicas y moleculares (LEAL-BERTIOLI, 1998; MARTINS 2005). O'Donnell et al., (2000); Summerell et al., (2003); Ottoni, (2008); comentar que con el advenimiento de herramientas moleculares la identificación de Fusarium se basa en la morfología, biología y característica de la especie, basada en la compatibilidad sexual, filogenia y secuencias de regiones genómicas.

Durante décadas, la identificación clásica utiliza características morfológicas como base, pero ha generado controversia, porque varios taxonomistas y fitopatólogos consideran necesario utilizar otros modelos para separar la especie, mientras que otros creen que estos modelos afectan la taxonomía del género. Cabe destacar que la práctica en el diagnóstico de agentes causales de enfermedades en la plantación de la adopción de modelos genéticos se adapta más a los estudios realizados por micólogos. Sin embargo, estos cambios son relevantes, ya que en un género puede haber especies muy cercanas y esto puede conducir a un diagnóstico erróneo con importantes consecuencias prácticas (QUERALES, 2010).

Según Notaro et al. (2013), Fusarium solani Math (Sacc) es la especie que causa pudrición de raíz en yuca cv branquinha en el estado de Pernambuco. Esta especie produce, en medio de cultivo, micelio aéreo, con un color que va del blanco a la crema, convirtiéndose en marrón azulado cuando hay esporoquios presentes. Produce tres tipos de esporas asexuales: la macroconidia en forma de puré, con dimensiones aproximadas de 15-35 x 4-7 m con 3-5 septa; microconidia, que tienen o no tabique, miden alrededor de 5-10 x 1-3 m y clamidosporas, que son hialina, globosa, lisa a áspera, que mide 6-10 m.

El estado sexual del hongo es Nectria haematococca (Ascomycete). Este hongo sobrevive a restos culturales infectados, suelo, tubérculos y semillas, y puede persistir durante varios años. Las condiciones ideales para su establecimiento son temperaturas que oscilan entre los 15 y los $250 \mathrm{C}$, la humedad del 50 al $75 \%$, la aparición de lesiones, la plantación en suelos contaminados y un almacenamiento inadecuado. Las lesiones en el sistema radicular, especialmente las causadas por nematodos, facilitan la penetración del hongo en las raíces de los huéspedes. 


\subsection{CONTROL DE ENFERMEDADES}

Debido a que es un patógeno transportado por el suelo, su eliminación después de la instalación en la zona de plantación se convierte en una tarea muy difícil y las medidas de control químico prácticamente no existen, excepto las preventivas, como el uso de tratamiento de semillas u otros materiales de propagación (LAZAROTTO et al., 2012). El control cultural, basado en la prevención de lesiones durante la cosecha y el almacenamiento, el uso de semillas sanas y sustrato libre del patógeno, utilizado una sola vez, la aplicación equilibrada de nutrientes, la eficiencia en el riego, así como las variedades que presentan características de resistencia y el tratamiento de esquejes (maniva) y sustrato con hongos de fungicidas para la siembra cuando se infectan cuando se infectan cuando se infectan cuando se infectan, puede minimizar las pérdidas de producción.

Como medida de control, la medida más eficiente se basó en investigaciones realizadas en el noreste por Embrapa Cassava y Fruit And Agricultural Development Company (EMAGRO), fue el uso de variedades tolerantes (Osso Duro, Cedinha, Bibiana, clon 148/02, Aramaris y Kiriris), asociada con el sistema de rotación y plantación de cultivos, que redujo la putrefacción en aproximadamente un $80 \%$ (GOMES; LEAL, 2003; BETTIOL, 2009; LOBO J-NIR et al., 2009; LAZAROTTO et al., 2012).

Para el control de Fusarium sp., el difenochonazol químico y el tiram, en el tratamiento del suelo y las semillas, son los más recomendados debido a la eficiencia presentada. Sin embargo, el control químico se ha vuelto constante y con el uso indiscriminado han surgido problemas ecológicos, ya que estos causan cambios en el medio ambiente favoreciendo fitopatógenos con resistencia debido a la presión selectiva, aparición de brotes secundarios, reducción de la población de microorganismos beneficiosos, debido al depósito de resid se acumula en el suelo, el agua y los alimentos haciéndolos dañinos, con efecto nocivo para animales y humanos (BIZI , 2015). 
En general, los elevados costos de la práctica del control químico, asociados con la aparición de resistencia a productos de uso común, así como la prohibición del bromuro de metilo, hacen que sea esencial desarrollar técnicas alternativas de control (BETTIOL, 2009; LOBO J-NIR et al., 2009; LAZAROTTO et al., 2012). Para la biológica, la inducción de la resistencia vegetal es una alternativa prometedora, ya que utiliza extractos naturales con propiedades microbianas y/o inductoras (MORAES, 1992; STANGARLIN et al., 2008).

\subsection{BIOCONTROL DE FITOPATÓGENOS}

El intento de reducir las acciones nociosas de un patógeno o inóculo ha sido una constante en la agricultura, y la sustitución de los agroquímicos por productos naturales con actividad de supresión o control ha sido una de las alternativas viables, ya que el residuo que queda es más rápidamente asimilado y descompuesto por el medio ambiente debido a su baja persistencia (SOUZA, 2013; BIZI, 2015).

Ethur, (2006) y Dianese, (2007); informar de la importancia de la investigación en el área del control biológico, como la introducción de organismos antagónicos en entornos donde el patógeno está causando daños. Cancela (2015), afirma que la dinámica de la acción del control biológico se produce de forma natural y consiste en la regulación del número de plantas y animales por parte de enemigos naturales. Esta estrategia es una parte fundamental del equilibrio de la naturaleza y de cómo el control químico tiene ventajas y desventajas. Entre las ventajas está el hecho de que se trata de una medida no tóxica, no causa desequilibrio, no tiene contraindicaciones, proporciona un control amplio y eficiente cuando no hay control químico, ya que necesitará más tecnología debido a su efecto lento, difícil de adquirir y no siempre se puede aplicar en cualquier época del año.

Mantener el equilibrio en el Agroecosistema es una práctica de control biológico para que el patosistema no cause daños significativos en la acción de biocontrol de organismos no patógenos del sistema. Los autores también informan que la enfermedad es más que una relación de interacción patógeno/huésped, porque la 
influencia del medio ambiente y también una diversidad de organismos no patógenos descansa en el lugar de la infección puede limitar o aumentar la agresividad del patógeno, o la resistencia del huésped. Así, patógenos, anfitriones y antagonistas interactúan entre sí en los componentes del control biológico (BIZI, 2015; CANCELA 2015).

Un factor limitante para el uso de productos biológicos es la disponibilidad en el mercado para esta modalidad de control. Otro problema es que muchos productos naturales no están debidamente registrados para su uso a escala comercial (SILVA et al., 2008). La presencia del fitopatógeno interfiere con la homeostasis vegetal, pero también puede actuar activando mecanismos de defensa y/o activando genes que confieren resistencia a la planta huésped (AGRIOS, 2005). Michereff et al., (1993), afirman que el patógeno tiene actividades determinantes que involucran crecimiento, infectividad, agresividad, virulencia y otras atribuciones del patógeno o procesos, que determinan la infección, el desarrollo de los síntomas y la reproducción. La interacción entre antagonista, huésped y patógeno no se produce de una manera particular, sino mutuamente.

Varios autores ya han descrito la capacidad de los biocontrangers para interactuar e interferir en el desarrollo del fitopatógeno mediante mecanismos de acción como la competencia por el espacio y nutrientes a la antibiosis, el micoparasitism o el hiperparasitismo, la depredación, la inducción de la resistencia, aumentando así la ventaja competitiva en el medio ambiente. Estos mecanismos varían de una especie a otra y también de linaje a linaje dentro de la misma especie, de acuerdo con la interacción huésped-parásito. (CARVALHO, 2006; BETTIOL; MORANDI, 2009; BRITO et al., 2010; ZUCCHI, 2010; DIAS, 2011; MACHADO et al., 2012; BIZI, 2015; CANCELA, 2015; FIPKE, et al., 2015).

Muchos microorganismos han presentado un alto potencial de biocontrolador y el género Trichoderma Pers. se ha utilizado en varios estudios. T. hamatum (Bonord.) Bainier, T. viride Pers., T. aureoviride Rifai, T. harzianum Rifai, $T$. koningii Oudem, $T$. pseudokoningii Rifai y T. longibrachiatum Rifai son los más citados (BETTIOL; GHINI, 
2005). Ezziyyani et al., (2007) informan que la frecuencia de Trichodema spp., se debe a su versatilidad ya sea por competencia, hiperparasitism y antibiosis a fitopatógenos como Phytophthora, Fusarium y Neoscytalidium. Remuska \& Pria (2007) evaluando el efecto antagónico de Trichoderma spp., en el control del crecimiento micelial de hongos fitopatógenos observó que esto ejerció antagonismo en algunos fitopatógenos radiculares, como Sclerotium rolfsii, Pythium aphanidermatum y Fusarium solani, este último género siendo descrito como uno de los principales fitopatógenos asociados con la podredumbre de la raíz de yuca en el estado de Pernambuco.

Hoffmann et al., (2015) informaron que tenían un 80\% de control de Fusarium sp., usando aislados de Trichoderma spp. Da Silva et al., (2011); Pereira (2013) demostró en sus obras un gran nivel de inhibición del crecimiento micelial de $F$. solani por trichoderma spp especies. Souza et al., (2015) también tuvieron resultados prometedores con Fusarium sp., y Bipolaris sp., usando el mismo biocontrolador. Otros estudios de Bomfim et al., (2010); Dias (2011); Lazarotto et al., (2012); Santos et al., (2012); Marques et al., (2014), obtuvieron resultados prometedores que presentan un buen rendimiento a excelente, con respecto a la inhibición del crecimiento micelial de fitopatógenos probados por ellos.

\subsection{ESTRATEGIAS DE BIOCONTROL DE TRICHODERMA}

El género Trichoderma, perteneciente al Reino de los Hongos, phylum Ascomycota, clases Eauscomycetes y Pyrenomycetes, orden Hypocreales, familia Hypocreaceae, género Hypocrea (teleomorfo). Este hongo tiene un gran potencial antagónico y a menudo se asocia con suelos supresores. (MELO, 1991). Según Harman et al. (2004); Harman (2005), su uso puede ofrecer otras ventajas: descomposición de materia orgánica, competencia con una microflora nociosa a través de la colonización de la rizosfera, producción de antibiosis e inducción de resistencia.

Las especies de Trichoderma ya han sido documentadas para el control de varios patógenos, tales como Rhizoctonia solani K-hn, Sclerotium rolfsii Sacc., Sclerotina 
sclerotiorum (Lib.) por Bary, Fusarium spp. e Pythium spp. (MELO; AZEVEDO, 1998). Numerosas especies de Trichoderma, incluyendo T. harzianum Hifai, mostraron una alta tasa de parasitismo y actividad antagónica para fitopatógenos como $\mathrm{S}$. rolfsii, $S$. minor y S. sclerotiorum, R. solani y Pyricularia grisea Sacc. (ROLLAN et al., 1999; RONDÓN et al., 2007).

Según Dos Santos (2010), el aislado de T. harzianum presentó antagonismo in vitro sobre el hongo S. rolfsii; ser capaz de hiperparasitizar las hifas del patógeno y competir por el espacio y los nutrientes. Silva et al. (2013), trabajando con aislados de Trichodema spp., como agente de control biológico de $S$. lignicola, observó que inicialmente el aislado de Neoscytalidium sp. fue muy prometedor cuando se enfrentó a Trichoderma spp., pero cuando fueron encontrados en el plato Petri, Trichoderma spp., superpuso la colonia de patógenos. Al observar la región donde el antagonista se superpone a los fitopatógenos, fue posible notar una degradación de las hifas del patógeno por acción enzimática, ya que se distinguió con precisión sólo las esporas y las hifas del antagonista.

Para Robbs (1992) y Barbosa; Meza (2009) la competencia por la alimentación y la antibiosis, son los mecanismos más utilizados por los agentes de biocontrol, ya que la reproducción y la colonización rápida son atributos de organismos que utilizan los recursos disponibles de manera más eficiente y rápida, asegurando su supervivencia. Según Ethur (2006), la competencia es una de las principales estrategias de trichoderma sp. aislados, por este hecho se utilizan como biocontroladores.

La secreción de sustancias antibióticas orgánicas (antibiosis), producidas por microorganismos, que incluso en baja concentración interfieren en las actividades metabólicas, la inhibición o la parada del crecimiento y la esporulación, la reducción de la germinación de las esporas y las distorsiones en las hifas y la endolisis, ya sean específicas o de amplio aspecto, son dispositivos importantes para la eficiencia de los organismos antagonistas (LOBO JUNIOR; ABREU, 2000; BOMFIM, 2007; SOUZA, 2013). Según Claydon et al., (1987), los antibióticos son productos del metabolismo secundario de sus productores, y pueden ser más importantes en la inhibición de otros 
organismos que la competencia por los nutrientes. La antibiosis también ha sido reportada por varios investigadores como uno de los mecanismos de acción de la especie Trichoderma (BETTIOL, 1991; DIAS, 2011; CARVALHO FILHO, 2013). Gliotoxina, viridina y tricodermina son algunas de las sustancias producidas por las especies de Trichoderma capaces de inhibir el desarrollo de otros hongos (Amorim et al., 2011). Ethur (2006) informó que la gliotoxina fue responsable de inhibir la germinación de los espomangios y el crecimiento micelial de Pythium ultimum.

Según Bomfim et al., (2010) y Souza (2013), el biocontrolador tendrá éxito si es capaz de degradar la pared celular (compuesta de carbohidratos, como la quitina), así como proteínas y lípidos en cantidades significativas del fitopatógeno o interrumpir los procesos relacionados con su síntesis. Por lo tanto, la producción de enzimas que hidrolizan los componentes de la pared celular de fitopatógenos, particularmente chitinasas y glucanasas, es una característica común en muchos agentes de biocontrol. Algunos representantes del yerno Trichoderma son citados en trabajos como un excelente productor de celulasa y chitinasas, enzimas que degradan la celulosa y la quitina (MELO, 1991; HARMAN et al., 2004).

El micoparasitismo, situación en la que el microorganismo antagonista vive en o dentro del hongo antagonizado, alimentándose y desarrollándose a su costa, es otro mecanismo importante de acción como atributo de un buen biocontrolador. El micoparasitism implica antibiosis y canibalismo causado por la acción de enzimas hidrolíticas, como chitinasas, glucanasas, proteasas y lipasas, que causan la muerte de una de ellas que por lo tanto sirve de alimento para el sobreviviente (LOBO JUNIOR; ABREU, 2000; HARMAN, 2000; BOMFIM, 2007; BARRA et al., 2008; ZUCCHI, 2010).

Las chitinasas y las glucanadas de 1,3-glucanés están directamente involucradas en las interacciones del micoparasitismo entre las especies de Trichoderma y sus huéspedes, debido a la mayoría de los hongos fitopatógenos que poseen en su pared celular de quitina, organizados en capas ordenadas regularmente y 1,3 glucanos dispuestos como un relleno de manera amorfa. La chitinasa actúa aleatoriamente y 
degrada las estructuras biológicas y los componentes de las células fúngicas, liberando oligómeros que inducen exoquitinas, iniciando así el ataque micoparasitario, hidrolizando los oligosacáridos de la pared celular del fitopatógeno. (GIESE et al., 2003; MARCELLO, 2008; BAUERMEISTER et al., 2010).

Melo (1996) informa que el micoparasitismo necrotrófico es eficiente en el antagonismo a los fitopatógenos, especialmente aquellos con estructuras de resistencia consideradas difíciles de ser atacados por microorganismos, como la espros, la esclerotia, las clamidosporas y la microesclerotia. Melo, melo. Azevedo (1998) afirma que Trichoderma sp. tiene características micoparásitos, porque pueden localizar y detectar fitopatógenos, posiblemente por estímulos bioquímicos, crecer hacia las hifas de hongos susceptibles, y cuando entran en contacto, forman estructuras como appressoriums, se acurrucan, en su totalidad, penetran y digieren. El género Trichoderma es sin duda un excelente agente de control microbiano, ya que presenta, además de las habilidades descritas anteriormente, características esenciales, siendo: impacto ambiental y bajo o nulo, fácil propagación (SPIEGEL; CHET, 1998), capacidad de sobrevivir en ambientes desfavorables, además de contener poblaciones de patógenos que viven en el suelo (VINALE et al., 2008).

\section{CONSIDERACIONES FINALES}

Las relaciones ecológicas interespecíficas son acontecimientos que ocurren a diversos niveles en la naturaleza y están hechos en su mayoría de formas demmónicas, en la condición de relación entre los microorganismos siendo la competencia, la antibiosis y el parasitismo más frecuentes. Por lo tanto, los microorganismos causantes de enfermedades en las plantas no sólo causan pérdidas económicas, sino que evolucionan simultáneamente, causando así daños desde la etapa inicial del desarrollo de los cultivos. Se han utilizado métodos tradicionales de control del fitopatógeno, la aplicación de pesticidas químicos y fungicidas, también ha sido una forma muy frecuente que causa problemas importantes debido a los impactos ambientales. 
Una de las formas más prometedoras de lograr una reducción en el uso de estos agroquímicos, o incluso una futura sustitución, control biológico a través de los numerosos biocontroladores como los hongos de/ género Trichoderma que son eficientes y no causan impactos ambientales destructivos.

\section{REFERENCIAS}

AGRIOS, G.N. Plant pathology. Amsterdam : Elsevier Academic Press. 5.ed, 2005. 922p.

ALVES, A., A., C.. Fisiologia da mandioca. In: Aspectos socioeconômicos e agronômicos da mandioca. EMBRAPA Mandioca e Fruticultura Tropical, Cruz das Almas, 2006., 817p.

AMORIM, L.; REZENDE, J. A. M.; BERGAMIN FILHO, A. Manual de Fitopatologia. Piracicaba: Agronômica Ceres. v. 1,4. Ed, p. 383 -387, 2011.

BARBOSA, R.J.F.; MEZA, C.L.S. Antagonismo in vitro de Trichoderma harzianum rifai sobre Fusarium oxysporum schlecht $f$. sp passiflorae en maracuyá (Passiflora edulis sims var. flavicarpa) del municipio zona bananera colombiana. Rev. Fac. Nal. Agr. Medellín, v.62, n.1, p.4743-4748, 2009.

BARRA, V. R.; ROMEIRO, R.S.; FERRAZ, H.G.M.; MACAGNAN, D.; SILVA, H. S. A.; MOURA, A. B.; HALFELD-VIEIRA, B. A; MENDONÇA, H. L.; VIEIRA JÚNIOR, J. R. Potencialidade antagonística em alguns procariotas agentes de biocontrole de enfermidades de plantas. Ver. Summa Phytopathologica, v.34, n.2, p.121-126, 2008.

BAUERMEISTER, A.; REZENDE, M. I.; GIESE, E. C.; DEKKER, R. F. H.; BARBOSA, A. M. 1,3-Glucanases Fúngicas: produção e aplicações biotecnológicas. LONDRINA SP. rev. Semina: Ciências Exatas e Tecnológicas, , v. 31, n. 2, p. 75-86, jul./dez. 2010. http://www.uel.br/seer/index.php/semexatas/article/view/5249 
BETTIOL, W. Controle biológico de doencas do filoplano. In: BETTIOL, W., org. Controle biológico de doencas de plantas. Jaguariuna: EMBRAPA-CNPDA, 1991. p.33-52 (EMBRAPA-CNPDA. Documentos, 15).

BETTIOL, W.: MORANDI, M.A.B. Controle Biológico de Doenças de Plantas no Brasil. In: BETTIOL, W.: MORANDI, M.A.B. (Org). Biocontrole de Doenças de Plantas: uso e perspectivas. Jaguariúna - SP: Embrapa Meio Ambiente, p. 7-14, 2009.

BETTIOL, W.; GHINI, R. Solos Supressivos. In: MICHEREFF,S. J.; ANDRADE, D. E. G. T.; MENEZES, M. (Eds.) Ecologia e Manejo de patógenos radiculares em solos tropicais. Recife:UFRPE, Imprensa Universitária, 2005. cap.6, p. 125-143.

BIZI, R. M., Utilização de fungos no Controle Biológico de Doenças. http://www.floresta.ufpr.br/alias/lpf/public_html/contbio03.html.> Acesso em 16/11/2015.

BOMFIM, M. P., Antagonismo in vitro e in vivo de Trichoderma spp. a Rhizopus stolonifer em maracujazeiro amarelo. Vitória da Conquista BA, 2007. 74f.: Dissertação de Mestrado. Universidade Estadual do Sudoeste da Bahia. 2007

BOMFIM, M. P., SÃO JOSÉ, A. R., REBOUÇAS, T. N. H., ALMEIDA, S. S. D., SOUZA, I. V. B., \& DIAS, N. O . Avaliação antagônica in vitro e in vivo de Trichoderma spp. a Rhizopusstolonifer em maracujazeiro amarelo. Summa Phytopathologyca, v.36, n.1, p. 61-67, 2010.

BRITO, F. S.; MILLER, P. R. M., STADNIK, M. Presença de Trichoderma spp em composto e suas características para o controle de fitopatógenos. Revista. Bras. de Agroecologia, Porto Alegre , p. 43-53. 2010.

CANCELA, K. C., Controle Biológico Florestal Conceitos, Terminologia, Descrição e Caracterização de Agente. Proteção florestal Disponível em $<$ http://www.floresta.ufpr.br/alias/lpf/public_html/contbio01.html> Acesso em 16/11/2015. 
CARVALHO FILHO, M. R., Relações filogenéticas, identificação e potencial de uso de isolados de Trichoderma no controle do mofo branco e como promotores de crescimento do feijoeiro. Brasília-DF, 2013. 123f. Tese de Doutorado. Programa de Pós-graduação em Fitopatologia, Universidade de Brasília. Brasília. 2013.

CARVALHO, A. C., Bioprospecção de isolados de Trichoderma stromaticum para o controle biológico da Vassoura-de-Bruxa do Cacaueiro. 2006. 82f. Dissertação de Mestrado. Universidade Estadual de Santa Cruz. 2006.

CONAB- CLAYDON, N.; ALLAN, M.; ITANSON, J.R.; AVENT, A. G. Antifungal alkyl pyrones of Trichoderma harzianum. Transactions of the British Mycological Society, v.88, p.503-513, 1987. COMPANHIA NACIONAL DE ABASTECIMENTO. Perspectivas para a agropecuária. Conab, Brasília , v.1, 2013., 154p.

DA SILVA, D. C. V., TIAGO, P. V., \& DE SOUZA-MOTTA, C. M. . Isolamento e seleção de fungos filamentosos do solo de sistemas agroflorestais do Município de Bom Jardim (PE) com base na capacidade de produção de enzimas hidrolíticas1. Revista Brasil. Bot, 34(4), 607-610. 2011

DIANESE, A. de C. Variabilidade e controle de Phytophthora palmivora (Podridão-do-pé) e controle da varíola (Aperisporium caricae) do mamoeiro (Carica papaya). 2007. 109f. Tese Doutorado - Universidade de Brasília, Brasília. 2007

DIAS, P. P., Controle biológico de fitopatógenos de solo por meio de isolados de fungos do gênero Trichoderma e sua contribuição no crescimento de plantas. 2011. 101 f. Tese Doutorado. Universidade Federal Rural do Rio de Janeiro. Seropédica - Rio de Janeiro. 2011

DINIZ, M. de S.. Efeito da manipueira na adubação da mandioca. Revista Raízes e Amidos Tropicais, v. 5, 2009. 
DOS SANTOS, J. et al. Atividade hiperparasíticade Trichoderma spp. sobre escleródios de Sclerotinia sclerotiorum em solo. In: SEMINÁRIO DE INICIAÇÃO CIENTÍFICA

TECNOLÓGICA,7,2010.Anais...EPAMIG,2010.Online.Disponívelem:http://www.epa mig.br/index.phpoption=com_docman\&task=cat_view\&gid=121\&dir=DESC\&order=da te\&limit=10\&limitstart=10.Acesso em Dezembro 2015

ETHUR, L.Z. Dinâmica populacional e ação de Trichoderma no controle de fusariose em mudas de tomateiro e pepino. 2006. 154p. Tese (Doutorado em Fitopatologia) - Universidade Federal de Santa Maria, Santa Maria-RS. 2006.

EZZIYYANI, M.; REQUENA, M. E.; EGEA-GILABERT, C.; CANDELA, M. E. Biological Control of Phytophthora Root Rot of Pepper Using Trichoderma harzianum and Streptomyces rochei in Combination. Journal of Phytopathology , [S.I], v.155, n.6, p. 342-349, 2007

FAO. Faostatidtics Database. Disponivel em <https://www.fao.org.br/FAO_Brf2mpu3a.asp> Acesso em Dezembro de 2015.

FIPKE, G. M; PAZINI, J.B.; ETHU,L. Z. Antagonismo de isolados de Trichoderma spp. ao Sclerotinia sclerotiorum em diferentes temperaturas. Magistra, Cruz das Almas BA, V. 27, N.1, p. 23 - 32, Jan./Mar. 2015

FUKUDA C.; OTSUBO A.A. Cultivo da mandioca na região centro sul do Brasil. Embrapa Mandioca e Fruticultura. HTTP://sistemasdeproducao.cnptia.embrapa.br/FontesHTML/Mandioca/mandioca_c entrosul/doenc s.htm . Acessado em 16 de julho de 2015.

FRAIFE FILHO G. A., BAHIA J. J. S., Mandioca. Ceplac-Cepec-BA. http://www.ceplac.gov.br/radar/mandioca.htm . Acessado Dezembro de 2015. 
GIESE, E. C.; BARBOSA, A. M.; SILVA M.L.C. Glucanases Fúngica: Produção e aplicações das -1,3 e -1,6 glucanases. Revista Biotecnologia Ciência e Desenvolvimento, Brasília-DF, № 30 , P.97-104.. jan/jun 2003.

GOMES, J. C.; LEAL, E. C., Cultivo da Mandioca para a Região dos Tabuleiros Costeiros. In.: Podridão Radicular. Embrapa Mandioca e Fruticultura. Sistemas de Produção, 11. ISSN 1678-8796 Versão eletrônica/Jan/2003. https://sistemasdeproducao.cnptia.embrapa.br/FontesHTML/Mandioca/mandioca_ta bcosteiros/doencas.htm . Acesso 13/12/2015.

HOFFMANN, C. A. et al.. Potencial de antagonismo de isolados de Trichoderma sp. contra o isolados de Fusarium sp., in vitro. Revista Verde de Agroecologia e Desenvolvimento Sustentável, v. 10, n. 1, p. 236-242, 2015.

HARMAN, G. E. The Nature and Application of Biocontrol Microbes II: Trichoderma spp. Overview of Mechanisms and Uses of Trichoderma spp. Phytopathology, St Paul, v. 96, p. 190-194. 2005

HARMAN, G. E. Myth and dogmas of biocontrol changes in perceptions derived from research on Trichoderma harzianum T-22. Plant Disease, St. Paul, v. 84, p.377-393, 2000.

HARMAN, G.E. HOWELL, C. R., VITERBO, A., CHET, I., \& LORITO, M. Trichoderma species-opportunistic, avirulent plant symbionts. Nature Reviews Microbiology. V.2, p. 43-56. 2004.

IBGE - Instituto Brasileiro de Geografia e estatística. PAS - Pesquisa Anual de Serviços, 2015. [online]Disponível na internet via WWW URL: http://www.ibge.gov.br/home/. Arquivo consultado Dezembro de 2015.

LAZAROTTO, M.; BOVOLINI, M. P.; MACIEL, C. G.; MUNIZ, M. F. B. Seleção in vitro de Isolados de Trichoderma spp. com Potencial de Antagonismo a Isolados 
Patogênicos de Fusarium spp. In: XVI Simpósio De Ensino, Pesquisa E Extensão, Unifra, v. 3, 2012.

LEAL- BERTIOLI, S. C. DE M. O enfoque molecular na sistemática de fungos. Revisão Anual de Patologia de Plantas. V.6. p. 197-230, 1998

LOBO JÚNIOR, M. e ABREU, M.S. Inibição do crescimento micelial de Sclerotinia sclerotiorum por metabólitos voláteis produzidos por alguns antagonistas em diferentes temperaturas e pH's. Ciência Agrotécnica, Lavras, v.24, p.521-526, 2000.

LOBO JUNIOR, M.; BRANDÃO, R. S.; CORRÊA, C. A.; GÖRGEN, C. A.; CIVARDI, E. A.; OLIVEIRA, P. de. Uso de braquiárias para o manejo de doenças causadas por patógenos habitantes do solo.Santo Antônio de Goiás: Embrapa Arroz e Feijão, 2009. 8 p. (Embrapa Arroz e Feijão. Comunicado técnico, 183).

MACHADO, D. F. M.; PARZIANELLO, F. R.; SILVA, A. C.F. e ANTONIOLLI, Z. I. Trichoderma no Brasil: o fungo e o bioagente. Revista de Ciências Agrárias, v.35, n.1, p. 274-288, 2012.

MARCELLO, C. M. Avaliação da expressão e caracterização de uma exo-b-1,3glucanase envolvida no mecanismo de micoparasitismo de Trichoderma asperellum. Brasília-DF, 2008. 89f. Tese de Doutorado. Laboratório de Enzimologia Departamento de Biologia Celular do Instituto de Biologia da Universidade de Brasília - UnB. Brasília - DF. 2008.

MARQUES, H. I. P.; SILVA, M. B.; MARQUES, M. D. P.; RODRIGUES, R. C.; RIBEIRO, P. R. C. C. Inibição do crescimento micelial de Trichoderma harzianum por fertilizantes líquidos. Enciclopédia Biosfera, v. 10, n. 18, p. 2040, 2014.

MARTINS, M. K.. Variabilidade genética de isolados de Fusarium spp. e estudo da interação com a planta hospedeira. 2005. 110f. Diss. Tese (Doutorado em Agronomia)-Escola Superior de Agricultura" Luiz de Queiroz", Universidade de São Paulo, SP.[Links], 2005 
MASSOLA JR, N.S.; BEDENDO, I. P.. Doenças da mandioca. In: KIMATI, H. et al. Manual de fitopatologia: Doenças das plantas cultivadas;. 4ª Ed.vol. 2, p. 340-341 São Paulo: Agronômica Ceres, 2005

MELO, I. S. Potencialidades da utilização de Trichoderma spp. no controle biológico de doenças de plantas. In: BETTIOL, W. (Ed.). Controle biológico de doenças de plantas. Jaguariúna: CNPDA/EMBRAPA, 1991. p. 135-156.

MELO, I. S.; AZEVEDO, J. L. Ecologia Microbiana. Jaguariúna: Embrapa DNPMA, p. 393-419, 1998.

MELO, I.S. Trichoderma e Gliocladium como bioprotetores de plantas. Revis. Anu. Patol. Plantas v. 4, p. 261-295. 1996.

MENEZES, J. P., LUPATINI, M., ANTONIOLLI, Z. I., BLUME, E., JUNGES, E., \& MANZONI, C. G. Variabilidade genética na região its do rDNA de isolados de trichoderma spp.(Biocontrolador) e Fusarium oxysporum f. sp. Chrysanthemi. Ciência e Agrotecnologia, 34(1), 132-139.2010

MICHEREFF, S.J.; MENEZES, M.; MARIANO, R.L.R. Potencial de Trichoderma para o controle da antracnose do sorgo. Fitopatologia Brasileira, Brasília, v. 18, p. 392-398, 1993.

MILANESI, P.M. Caracterização, toxicidade e patogenicidade de Fusarium spp. em genótipos de soja em sistema plantio direto. 2009. 91 p. Dissertação (Mestrado em Agronomia) - Universidade Federal de Santa Maria, Santa Maria, 2009.

MORAES, W. B. C. Controle alternativo de fitopatógenos. Pesquisa Agropecuária Brasileira, v. 27, n. 13, p. 175-190, 1992.

MORAIS, M. dos S.; NASCIMENTO, L. C do; MOREIRA, K. A.; SILVA, M. da; CAVALCANTI, N. T. D. O. Levantamento e avaliação da incidência das doenças da mandioca no estado da Paraíba. Summa Phytopathologica, v. 39, n. 3, p. 204. 2013. 
MOURA, G. de M.; SILVA, M. D. O. da. Avaliação de resistência de cultivares de mandioca à podridão de raízes. Embrapa-CPAF/AC. Comunicado Técnico, 4p. Rio Branco 1997.

MUNIZ J. N, RUFFINO-NETTO A, YAMAMURA M, VILLA TCS, ARCENCIO R. Aspectos epidemiológicos da coinfecção tuberculose e vírus da imunodeficiência humana em Ribeirão Preto (SP), de 1998 a 2003. J. Bras. Pneumol., 32(6):529-34, 2006

NASCIMENTO JÚNIOR, N. A. Efeito da casca de mandioca no controle da podridão radicular causada por Phytophthora sp. em mandioca de mesa (Manihot esculenta Crantz) var. Rosinha em ambiente irrigado. Rio LargoAL,2015.82f. Tese de Doutorado. (Programa de Pós-Graduação em Proteção de Plantas da Universidade Federal de Alagoas. Alagoas. 2015.

NOTARO, K. A. MEDEIROS, E. V., SILVA, C. A. D., \& Barros, J. A. Prospecção de fitopatógenos associados á podridão radicular da mandioca em Pernambuco, Brasil. Biosci. J., Uberlândia, v. 29, n. 5, p. 1832-1839, 2013.

NOTARO, K. A. Prospecção de fitopatógenos e caracterização de solos arenosos envolvidos na supressividade ou conducividade da podridão radicular da mandioca, causada por Neoscytalidium lignicola/ Krystal de Alcantara Notaro. Garanhuns-PE, 2012. 111f. Dissertação (PROGRAMA DE PÓS-GRADUAÇÃO EM PRODUÇÃO AGRÍCOLA). Universidade Federal Rural de Pernambuco - Unidade Acadêmica de Garanhuns, 2012.

O`DONNELL, K.; NIRENBERG, H.I.; AOKI, T.; CIGELNIK, E. A multigene phylogeny of the Gibberella fujikoroi species complex: detection of additional phylogenetically distinct species.Mycoscience, Tokyo, v. 41, p. 61-78, 2000.

OLIVEIRA, L. G. SILVA, A.C.S; SILVA, R. L. ,LIMA, D. W. S \& LIMA, G; COSTA. Atividade proteolítica de fungos de solo fitopatogênicos ao Feijão-Caupi [Vigna 
unguiculata (L.) Walp.] In: III CONAC Congresso Nacional de Feijão-Caupi. Pernambuco. Resumo expandido. 4p. 22-24 de abril Recife Pernambuco 2013.

OLIVEIRA, N. T; J. M. A., UCHÔA, S. C. P., RODRIGUES, G. S., MELVILLE, C. C., \& de ALBUQUERQUE, J. D. A. A.. Caracterização e identificação de clones de mandioca produzidas em Roraima para o consumo in natura. Revista Agro@mbiente On-line, v. 5, n. 3, p. 188-193, 2012.

OTTONI, R.J. Analise da incidência de Fusarium spp. toxigenicos e de níveis de fumonisinas em grãos ardidos de milho hibrido. 2008. 54 p. Dissertação (Mestrado em Microbiologia Agrícola) - Escola Superior de Agricultura "Luiz de Queiroz", Universidade de São Paulo, Piracicaba, 2008.

PEREIRA, C O.F. Estudo da patogenicidade e controle biológico de Fusarium sp. Com Trichoderma sp. 2013.74f. Dissertação. Universidade de Caxias do Sul. 2013.

PUHALLA, J.E. Genetic considerations of the genus Fusarium. In: Nelson, P.E.; Toussoun, T.A.; Cook, R.J. (Ed) Fusarium: diseases, biology, and taxonomy. Pennsylvania: Pennsylvania State University, v.27, p.291-305, 1981.

QUERALES, P. J.. Caracterização morfológica e genética de Fusarium spp. isolados de sementes e associados à podridão do colmo de milho (Zea mays L.). Diss. Escola Superior de Agricultura "Luiz de Queiroz, 2010.

REMUSKA, A.C.; DELLA PRIA, M. Efeito de Bacillus truringiensis e Trichoderma sp. no crescimento de fungos fitopatogênicos. Publication UEPG: Ciências Exatas e da Terra, Agrárias e Engenharia, Ponta Grossa, v.13, n.3, p.31-36, 2007.

ROBBS, F. C. Controle Biológico de doenças em plantas. In: NETO, A. M. A.; BARAN, C. L. (ed.). Manual de Controle Biológico. Rio de Janeiro: Lidador. 1992. p.4651. 
ROLLAN, MONACO C, NICO A. Efecto de la tempratura sobre la interaccion invitr o entre especies de Tricihoderma y Sclerotinia sclerotiorum, S. minor y Sclerotium rolfsii. Invest. Agr.: Prod. Prot. Veg. 14:1-16. 1999.

RONDÓN T.R, GUTIÉRREZ GR, ZAYAS A.D.P, PÉREZ LA, CUTIÑO Y.L. Efectividad in vitro de Trichoderma harzianum Rifai para el biocontrol de Rhizoctonia solani Kühn y Pyricularia Grisea sacc. aislados en el cultivo del arroz (Oryza sativa I.). Fitosanidad 11: 29-34.2007.

SALLA, D. A., CABELLO, C. Análise energética de sistemas de produção de etanol de mandioca, cana-de-açúcar e milho. Revista Energia na Agricultura. Botucatu, vol. 25, n.2, p.32-53., 2010.

SANTOS, C. C., OLIVEIRA, F. A. de; SANTOS, M. S. dos; TALAMINI, V.; FERREIRA, J. M. S.; SANTOS, F. J. dos., Influência de Trichoderma spp. sobre o crescimento micelial de Thielaviopsis paradoxa. Scientia Plena 8, 047309 www.scientiaplena.org.br . 2012

SEIFERT, K.A. Enhancing the relevance of fungal taxonomy to plant pathology: phylogenetics, molecular diagnostics, and long-term memory1. Symposium contribution. Canadian Journal of Plant Pathology, Otawa, v. 28, p. S280-S287, 2006.

SILVA, A. N.; AZEVEDO, G. B.; ROCHA-SOBRINHO, G. G.; NOVAES, Q. S.; Efeito de produtos químicos e de Trichoderma spp. no controle de Fusarium solani do maracujazeiro. Interciencia, v. 6, p. 398-403, 2014.

SILVA, C. A. D., DE MEDEIROS, E. V., BEZERRA, C. B., DE MORAIS; SILVA, W., DE BARROS, J. A., \& DOS SANTOS, U. J. Interferência da incorporação de matéria orgânica no solo no controle da podridão negra da mandioca, causada por Scytalidium lignicola. Biosci. J., Uberlândia, v. 29, n. 6 , p. 1823-1831, Nov./Dec. 2013. 
SILVA, C. A. D., Prospecção em fitopatógenicos e avaliação de fontes de matéria orgânica sobre a supressividade da podridão radicular da mandioca. GaranhunsPE, 2013. 77 f. Dissertação (Mestrado em Produção Agrícola). Universidade Federal Rural de Pernambuco - Unidade Acadêmica de Garanhuns, 2013.

SILVA, H. S. A.; ANDRADE, E. C. Impacto potencial das mudanças climáticas sobre as doenças da mandioca no Brasil. In: Impactos das mudanças climáticas sobre doenças de importantes culturas no Brasil. Jaguariúna: Embrapa Meio Ambiente, p. 263-272, 2011.

SILVA, J. L.; TEIXEIRA, R. N. V. Esporulação e crescimento micelial de Fusarium solani em diferentes meios de cultura e regimes de luminosidade. Revista Agrombiente On-line,v.6,n.1,p.47-52, 2012. Disponível em: $<$ <ttp://revista.ufrr.br/index.php/agroambiente/article/view/604>

SILVA, K. S.; REBOUÇAS, T. N. H.; BOMFIM, M. P.; SILVA, D. S.; SÃO JOSÉ, A. R.; BENETT, C. G. S. Atividade antagônica in vitro de isolados de Trichoderma spp. ao fungo Phytophthora citrophthora. Londrina. Semina: Ciências Agrárias, v. 29, n. 4, p. 749-754, out./dez. 2008.

SILVA, M.B.; NICOLI, A. COSTA, A.S.V.; BRASILEIRO, B.G.; JAMAL, C.M., SILVA, C. A.; PAULA JÚNIOR, T. J.; TEIXEIRA, H. Ação antimicrobiana de extratos de plantas medicinais sobre espécies fitopatogênicas de fungos do gênero Colletotrichum. Revista Brasileira de Plantas Medicinais, Botucatu, v.10, n.3, p.5760, 2008.

SOUSA, R.M.S.; SERRA, I.M.R.S.; MELO, T.A. Effect of essential oils as an alternative in the control of Colletotrichum gloesporioides in pepper.Summa Phytopathologica, v.38, n.1, p.42-47, 2012.

SOUZA, J. R., Potencialidade de fungicida e agente biológico no controle de requeima do tomateiro. 2013. 63f. Dissertação. Universidade Estadual do Sudoeste da Bahia, Vitória da Conquista. Bahia - BR. 2013. 
SOUZA, K. de A.;VAZ, M. C. A.; ALVES, G. C. S.; PAZ -LIMA, M. L.. Confronto in vitro de isolados de Trichoderma spp. com fungos filamentosos. 2p. IV Congresso Estadual de Iniciação Científica do IF Goiano 21 a 24 de setembro de 2015.

SPIEGEL, Y.; CHET, I. Evolution of Trichoderma spp. as a biocontrol agent against soilborne fungi and plant parasitic nematodes in Israel. Integrated Pest Management Review, v. 03, p. 167-175, 1998.

STANGARLIN, J.R. et al. Controle de doenças de plantas por extratos de origem vegetal. Revisão Anual de Patologia de Plantas, v.16, p.265-304, 2008.

URBEN, A.F., et al., Curso taxonomia de Fusarium. Embrapa recursos genéticos e biotecnologia. Brasilia-DF: Embrapa informação tecnólogica, 2009.

VENTURA, J. A. Taxonomia de Fusarium e seus segregados: I- história, meios e procedimentos de cultivo. Revisão Anual de Patologia de Plantas, Passo Fundo, v. 7, p. 271-298, 1999.

VINALE F, SIVASITHAMPARAM, K., GHISALBERTI, E. L., MARRA, R., WOO, S. L., \& LORITO, M.. Trichoderma-plant-pathogen interactions. Soil Biology and Biochemistry, v. 40, p.1-10.2008.

ZUCCHI, F. O. Trichoderma sp. em áreas agrícolas visando o controle de doenças fúngicas de raízes de plantas cultivadas. Campinas SP. JV Biotecnologia. 15p. 2010.

\section{APÊNDICE - REFERÊNCIA DE NOTA DE RODAPÉ}

1. WOLLENWEBER, H. W.; REINKING, O. A.. Die fusarien: ihre beschreibung, schadwirkung und bekämpfung. Berlin: P. Parey, 1935

Enviado: Mayo, 2020.

Aprobado: Junio de 2020. 